\title{
Lipocalin 2 regulates retinoic acid-induced activation of beige adipocytes
}

\author{
Jessica A Deis', Hong Guo', Yingjie Wu², Chengyu Liư3, David A Bernlohr ${ }^{4}$ and Xiaoli Chen' \\ 1Department of Food Science and Nutrition, University of Minnesota, Twin Cities, Minnesota, USA \\ 2Institute for Genomic Engineered Animal Models of Human Diseases, Dalian Medical University, Dalian, China \\ ${ }^{3}$ Transgenic Core, National Heart, Lung and Blood Institute, National Institutes of Health, Bethesda, Maryland, USA \\ ${ }^{4}$ Department of Biochemistry, Molecular Biology and Biophysics, University of Minnesota, Twin Cities, Minnesota, USA \\ Correspondence should be addressed to X Chen: xlchen@umn.edu
}

\begin{abstract}
Lipocalin-2 (LCN2) has been previously characterized as an adipokine regulating thermogenic activation of brown adipose tissue and retinoic acid (RA)-induced thermogenesis in mice. The objective of this study was to explore the role and mechanism for LCN2 in the recruitment and retinoic acid-induced activation of brown-like or 'beige' adipocytes. We found LCN2 deficiency reduces key markers of thermogenesis including uncoupling protein-1 (UCP1) and peroxisome proliferator-activated receptor gamma coactivator $1 \alpha$ (PGC-1 $\alpha$ ) in inguinal white adipose tissue (iWAT) and inguinal adipocytes derived from $L c n 2^{-/-}$mice. $L c n 2^{-I-}$ inguinal adipocytes have attenuated insulin-induced upregulation of thermogenic gene expression and p38 mitogen-activated protein kinase (p38MAPK) signaling pathway activation. This is accompanied by a lower basal and maximal oxidative capacity in Lcn2-l- inguinal adipocytes, indicating mitochondrial dysfunction. Recombinant LCN2 was able to restore insulin-induced p38MAPK phosphorylation in both WT and $L c n 2^{-/-}$inguinal adipocytes. Rosiglitazone treatment during differentiation of $L c n 2^{-1-}$ adipocytes is able to recruit beige adipocytes at a normal level, however, further activation of beige adipocytes by insulin and RA is impaired in the absence of LCN2. Further, the synergistic effect of insulin and RA on UCP1 and PGC-1 $\alpha$ expression is markedly reduced in Lcn $2^{-1-}$ inguinal adipocytes. Most intriguingly, LCN2 and the retinoic acid receptor-alpha (RAR- $\alpha$ ) are concurrently translocated to the plasma membrane of adipocytes in response to insulin, and this insulin-induced RAR- $\alpha$ translocation is absent in adipocytes deficient in LCN2. Our data suggest a novel LCN2mediated pathway by which RA and insulin synergistically regulates activation of beige adipocytes via a non-genomic pathway of RA action.
\end{abstract}
Key Words
- lipocalin 2
- adipocyte
- thermogenesis
retinoic acid
- insulin

Journal of Molecular Endocrinology (2018) 61, 115-126

\section{Introduction}

Obesity prevalence continues to increase along with escalating rates of metabolic diseases including diabetes and cardiovascular disease (Flegal et al. 2016). Thermogenic activity in adipose tissue is associated with a decreased BMI and improved metabolic health (van
Marken Lichtenbelt et al. 2009, Harms \& Seale 2013). Although the presence of brown adipose tissue (BAT) in adult humans has been confirmed, the BAT established in infancy dramatically decreases with age (Saito et al. 2009). Therefore, the conversion of energy-storing white adipose 
tissue (WAT) to energy expending BAT holds therapeutic potential. White adipocytes can take on a brown-like, or 'beige' phenotype, characterized by increased number of mitochondria, a multilocular appearance, expression of uncoupling protein-1 (UCP1)-positive cells and a functional increase in energy expenditure (Ohno et al. 2012, Harms \& Seale 2013).

Several factors are known to promote beiging of WAT, including cold, dietary factors, exercise, pharmaceuticals and circulating factors including adipokines (Harms \& Seale 2013). Thermogenesis is classically activated by catecholamines released during cold exposure, which bind to $\beta$-adrenergic receptors on the surface of adipocytes. This activates a protein kinase A, p38 mitogen-activated protein kinase (p38MAPK), activating transcription factor-2 (ATF2) signaling cascade, which concurrently upregulates mitochondrial biogenesis, the expression of thermogenic genes and lipolysis to provide fatty acids to support uncoupling of the electron transport chain (Cao et al. 2001, 2004). However, alternative, non-adrenergic pathways have also been identified as promoting beiging of WAT.

The anti-diabetic drug rosiglitazone acts as a peroxisome proliferator-activated receptor $\gamma$ (PPAR $\gamma$ ) agonist and upregulates transcription of mitochondrial and thermogenic genes including Ucp1 (Petrovic et al. 2008, 2010, Ohno et al. 2012). Chronic treatment of WAT with rosiglitazone recruits beige adipocytes, but is not by itself able to activate thermogenesis without further stimulation by norepinephrine (Nedergaard \& Cannon 2014). It is unclear whether there are endogenous factors that act in a similar manner to rosiglitazone, as well as whether non-adrenergic beiging activators require or influence PPAR $\gamma$-mediated recruitment of beige adipocytes.

RA, a known activator of BAT thermogenesis, binds to and promotes nuclear translocation of transcription factors that upregulate Ucp1 gene expression (Alvarez et al. 1995, Rabelo et al. 1996, Bonet et al. 1997, Kumar et al. 1999). $\mathrm{RA}$ is also reported to increase p38MAPK phosphorylation, which was found necessary for the RA-induced upregulation of Ucp1 gene expression (Teruel et al. 2003, Mercader et al. 2010). Therefore, it is possible that RA regulates thermogenesis through multiple pathways, although the exact mechanism is unknown.

Lipocalin-2 (LCN2) is a $25 \mathrm{kDa}$ secreted protein expressed highly in adipose tissue depots in response to cold and inflammatory stimulation (Guo et al. 2010, Zhang et al. 2014a). Solution structures of LCN2 indicate it has an anti-parallel $\beta$-barrel structure, which surrounds a hydrophobic-binding pocket (Coles et al. 1999). This structure is conserved among the lipocalin family and allows them to bind to a variety of small, hydrophobic molecules such as fatty acids, retinoids and steroids (Flower 1996). We have previously been shown that Lcn2-/mice are not able to maintain their body temperature when exposed to cold (Guo et al. 2010). Lcn2-1- mice developed significantly increased body fat mass, adipocyte hypertrophy and adipose inflammation compared to WT mice upon a high-fat diet (HFD) feeding (Guo et al. 2010). Subsequent examination of BAT in Lcn2-l- mice found deficiencies in induction of thermogenic genes and mitochondrial biogenesis following cold exposure (Zhang et al. 2014b). Conversely, catecholamine levels and the response to $\beta$-adrenergic activators are normal in Lcn2-/- mice and brown adipocytes, suggesting LCN2 regulates thermogenesis through a non-adrenergic mechanism (Zhang et al. 2014b). Lcn2-/- mice have decreased expression of PPAR $\gamma$ in brown and WAT depots and chronic treatment with rosiglitazone has been shown to improve insulin sensitivity and cold intolerance in these mice (Jin et al. 2011). Further studies have found LCN2 regulates RA metabolism and action in adipose tissue (Guo et al. 2016). These previous studies suggest that LCN2 may regulate RA-induced thermogenesis in adipose tissue. However, the exact pathway by which LCN2 regulates adipose tissue, especially beige adipose tissue, thermogenesis remains unknown. Therefore, the objective of this study was to determine the role of LCN2 in the regulation of beiging of white adipocytes and to identify a non-adrenergic pathway that is responsible for LCN2 regulation of RA-induced activation of beige adipocytes.

\section{Materials and methods}

\section{Animals}

LCN2-deficient mice were kindly provided by Dr. Alan Aderem, Institute for Systems Biology, Seattle, Washington, USA. Heterozygous mating scheme was used to generate WT and Lcn $2^{-/-}$mice as previously described (Guo et al. 2010). Animals were housed at $22^{\circ} \mathrm{C}$ in a specific pathogen-free facility at the University of Minnesota. Animal studies were conducted with the approval of the University of Minnesota Animal Care and Use Committee and conformed to the National Institute of Health guidelines for laboratory animal care.

For in vivo studies with RA treatment, WT and Lcn $2^{-/-}$mice from the same litter were housed at $22^{\circ} \mathrm{C}$ in a 12:12-h light-darkness cycle with free access to water. Male mice were fed a HFD (60\% of calories from fat, Bioserv F3282, New Brunswick, NJ, USA) at 4 weeks of age for 
12 weeks. At 16 weeks of age, three male WT and five male Lcn2-/- mice were treated with $50 \mathrm{mg} / \mathrm{kg}$ body weight alltrans RA (ATRA, Sigma) via oral gavage daily for 24 days. Control mice were treated with $1 \%$ ethanol vehicle. HFD was continued during the 24-day treatment period. After 24-day treatment, tissues were collected and immediately frozen in liquid nitrogen and stored at $-80^{\circ} \mathrm{C}$ for use.

\section{Cell culture and differentiation of primary stromal-vascular cells}

Stromal-vascular (SV) cell fraction containing preadipocytes were isolated from inguinal WAT of WT and Lcn2-I- male mice as previously described (Zhang et al. $2014 b$ ). Cells were grown to confluence in Dulbecco's Modified Eagle Medium (DMEM; Invitrogen) containing L-glutamineand 25 mMglucose, 10\% FBS (Atlanta Biological, Lawrenceville, GA, USA) and 100 units/mL penicillin/ streptomycin (Invitrogen). After reaching confluence, pre-adipocytes were treated with a differentiation cocktail for 2 days containing DMEM, 10\% FBS, penicillin/ streptomycin, $0.25 \mathrm{mM}$ isobutylmethylxanthine (Sigma), $0.5 \mu \mathrm{M}$ dexamethasone (Sigma) and $0.85 \mu \mathrm{M}$ insulin. The cells were then maintained in culture media with $0.85 \mu \mathrm{M}$ insulin until fully differentiated. For rosiglitazone experiments, $1 \mu \mathrm{M}$ rosiglitazone (Sigma) was added to the culture media from day 0 of differentiation until fully differentiated. For insulin stimulation, differentiated adipocytes were serum starved in DMEM containing 0.5\% FBS for 3-6h prior to addition of insulin. Recombinant Lcn2 was commercially obtained from R\&D Systems.

\section{Plasma membrane isolation}

For the preparation of crude plasma membrane fractions, a previously described method was followed (Chen et al. 2003). Briefly, differentiated primary adipocytes were harvested and homogenized in TES buffer containing $20 \mathrm{mM}$ Tris, $1 \mathrm{mM}$ EDTA and sucrose. Homogenates were layered onto a sucrose cushion and centrifuged at $100,000 \boldsymbol{g}$ for 20 minutes in a SW60 Ti swinging bucket rotor. Interface was collected and centrifuged at $130,000 \mathrm{~g}$ for $10 \mathrm{~min}$ in a TLA120.2 rotor to obtain the final pellet containing the plasma membrane fraction.

\section{Statistical analysis}

Values are reported as mean \pm S.E.M.. Statistical significance was determined by two-tailed Student's $t$ test, where a $P$ value less than 0.05 was considered significant.

\section{Results}

LCN2 deficiency impairs beiging and mitochondrial function in Lcn2-/- inguinal adipose tissue

Lcn2 gene expression and protein levels in inguinal WAT (iWAT) are increased in response to thermogenic stimuli, including cold exposure and treatment with the $\beta$-adrenergic agonist norepinephrine (NE) (Guo et al. 2010, Zhang et al. 2014a). We therefore sought to determine a role for LCN2 in WAT beiging. We found Ucp1 expression was significantly attenuated in iWAT from $L c n 2^{-l-}$ mice, signifying possible impairments in beiging in the absence of LCN2 (Fig. 1A). This effect was not seen in epididymal WAT (eWAT), which has previously been reported to have less thermogenic capacity than iWAT (Walden et al. 2012). Further, primary adipocytes isolated from iWAT from Lcn $2^{-/-}$mice showed significantly attenuated expression of thermogenic genes $U c p 1$ and peroxisome proliferatoractivated receptor gamma coactivator $1 \alpha(P g c 1 a)$ compared to those from WT mice (Fig. 1B), suggesting impairment in beiging capacity.

Since beiging capacity is positively correlated with mitochondrial function (Wu et al. 2012), we next looked at functional measurements of mitochondrial activity. Mitochondrial respiratory profile analysis by Seahorse XF Analyzers showed that oxygen consumption rates were significantly decreased in $L c n 2^{-/-}$differentiated inguinal adipocytes under both basal and maximal respiration conditions, indicating a decrease in mitochondrial oxidative capacity (Fig. 1C and D). Consistent with decreased mitochondrial function, $\mathrm{NAD} / \mathrm{NADH}+$ ratio was decreased in Lcn2-I- inguinal adipocytes under both fed and starved conditions (Fig. 1E). Mitochondrial DNA copy number was similar between WT and Lcn2-I- inguinal adipocytes (Fig. 1F), suggesting that mitochondrial content may not be affected. However, further investigation would be needed in order to make a solid conclusion on the effect of Lcn2 $\mathrm{KO}$ on mitochondrial biogenesis and number. Taken together, these data suggest adipocytes deficient in LCN2 have reduced beiging capacity and impaired mitochondrial function.

\section{LCN2 deficiency does not affect $\beta$-adrenergic signaling in inguinal adipocytes}

To test the impact of LCN2 deficiency on $\beta$-adrenergic signaling in adipocytes, SV cells isolated from WT and Lcn2-- iWAT were differentiated to mature adipocytes. We treated differentiated WT and $L c n 2^{--}$- inguinal adipocytes with norepinephrine (NE) for $1 \mathrm{~h}$. Phosphorylation 

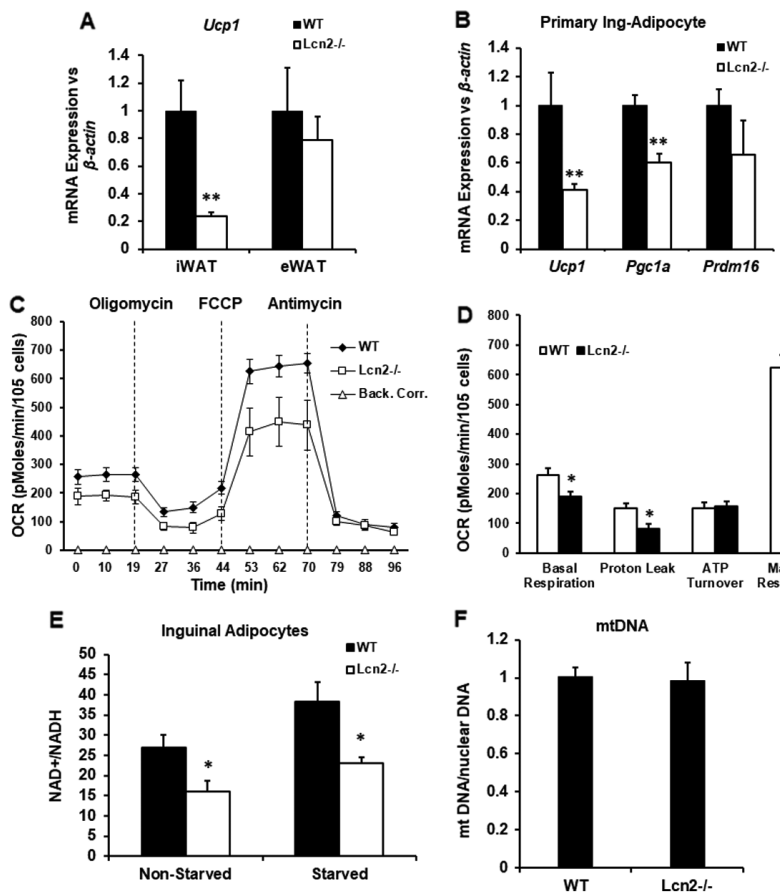

Figure 1

Thermogenic gene expression and mitochondrial function in Lcn2-I- WAT and inguinal adipocytes. (A) Ucp1 gene expression in inguinal WAT (iWAT) and epididymal WAT (eWAT) from WT and $L C n 2^{-1-}$ mice on high-fat diet for 12 weeks, $n=3-5 /$ group. (B) Thermogenic gene expression in primary inguinal adipocytes isolated from WT and LCn2--- mice, $n=3-5 /$ group. (C and D) Oxygen consumption rate in differentiated inguinal adipocytes isolated from WT and $L \mathrm{Cn} 2^{-l-}$ mice, $n=8 /$ treatment. (E) NAD+/NADH in differentiated inguinal adipocytes isolated from WT and $L C n 2^{---}$mice, $n=3 /$ treatment. (F) mtDNA relative to nuclear DNA in inguinal differentiated adipocytes from WT and $L \mathrm{Cn} 2^{---}$mice, $n=3 /$ treatment. The cell culture experiments were repeated 2-3 times yielding similar results. The data are represented as mean \pm s.E.M. ${ }^{*} P<0.05$ vs WT; ${ }^{*} P<0.01$ vs WT.

of p38MAPK in response to acute NE treatment was similar between WT and Lcn2-l- adipocytes, indicating $\beta$-adrenergic signaling is intact in the absence of LCN2 (Fig. 2A and B). Similarly, there was no significant difference in induction of $U c p 1$ gene expression by $\mathrm{NE}$ in $L \mathrm{cn} 2^{-/-}$differentiated inguinal adipocytes when compared to WT (Fig. 2F). As insulin-induced thermogenic signaling and gene expression is impaired in the absence of Lcn2, we also tested $\beta$-adrenergic signaling in the presence of insulin. However, NE-induced phosphorylation of p38MAPK remained higher in Lcn2-/- adipocytes despite the addition of insulin (Fig. 2A and B). Similar to p38MAPK, phosphorylation of hormone-sensitive lipase (HSL) in response to acute NE treatment was higher in Lcn2-/- inguinal adipocytes when compared to WT adipocytes (Fig. 2C and D). Next, we treated differentiated inguinal adipocytes with isoproterenol and measured glycerol release as an indicator of $\beta$-adrenergically
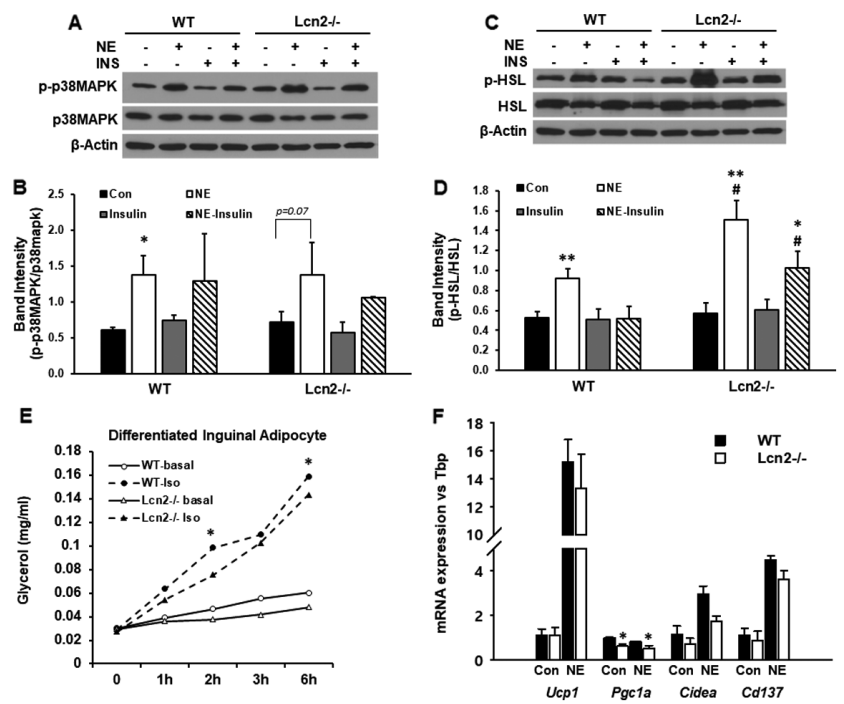

Figure 2

$\beta$-adrenergic signaling in Lcn2-- inguinal adipocytes. p38MAPK (A and B) and HSL (C and D) phosphorylation following treatment with $1 \mu \mathrm{M}$ norepinephrine $(\mathrm{NE}) \pm$ insulin (Ins). (E) Glycerol release into cell supernatant from differentiated inguinal adipocytes treated with $1 \mu \mathrm{M}$ isoproterenol (Iso). (F) Gene expression in differentiated inguinal adipocytes treated with $1 \mu \mathrm{M}$ norepinephrine (NE) for $18 \mathrm{~h}, n=3$ / treatment. Experiments were repeated two times yielding similar results. The data are represented as mean \pm S.E.M. For $B$ and $D, * P<0.05$ vs Control of same genotype; ${ }^{*} P<0.01$ vs Control of same genotype; ${ }^{*} P<0.01$ vs WT of same group. For $E$ and $F, * P<0.05$ vs WT of same group.

activated lipolysis. Isoproterenol was able to induce glycerol release in $L c n 2^{-/-}$inguinal adipocytes, although this was slightly decreased when compared to WT inguinal adipocytes (Fig. 2E). Together, these data points toward a non-adrenergic mechanism by which LCN2 regulates thermogenic signaling in inguinal WAT.

\section{LCN2 regulates p38MAPK signaling pathway activation and thermogenic gene expression in inguinal adipocytes}

As p38MAPK signaling is a major upstream pathway regulating thermogenic gene expression (Cao et al. 2001, 2004), we next investigated whether LCN2 regulates this thermogenic pathway in inguinal adipocytes. First, we examined the effect of Lcn2 deficiency on the Ucp1 and Pgc1 $\alpha$ expression in differentiated inguinal adipocytes. After 18-h culture in the presence or absence of insulin, the expression of Ucp1, Pgc1a and Cidea genes was increased by insulin stimulation in WT adipocytes (Fig. 3A). Interestingly, the stimulatory effect of insulin on thermogenic gene expression was significantly attenuated in $L c n 2^{-/}$adipocytes (Fig. 3A). Next, differentiated adipocytes were treated with recombinant LCN2 in 
the absence or presence of insulin for $18 \mathrm{~h}$ to examine the effects of LCN2 on p38MAPK signaling. There was no statistically significant difference in basal levels of p38MAPK phosphorylation between WT and Lcn2-Iadipocytes (Fig. 3B and C). After 18-h treatment, insulin significantly increased p38MAPK phosphorylation in WT

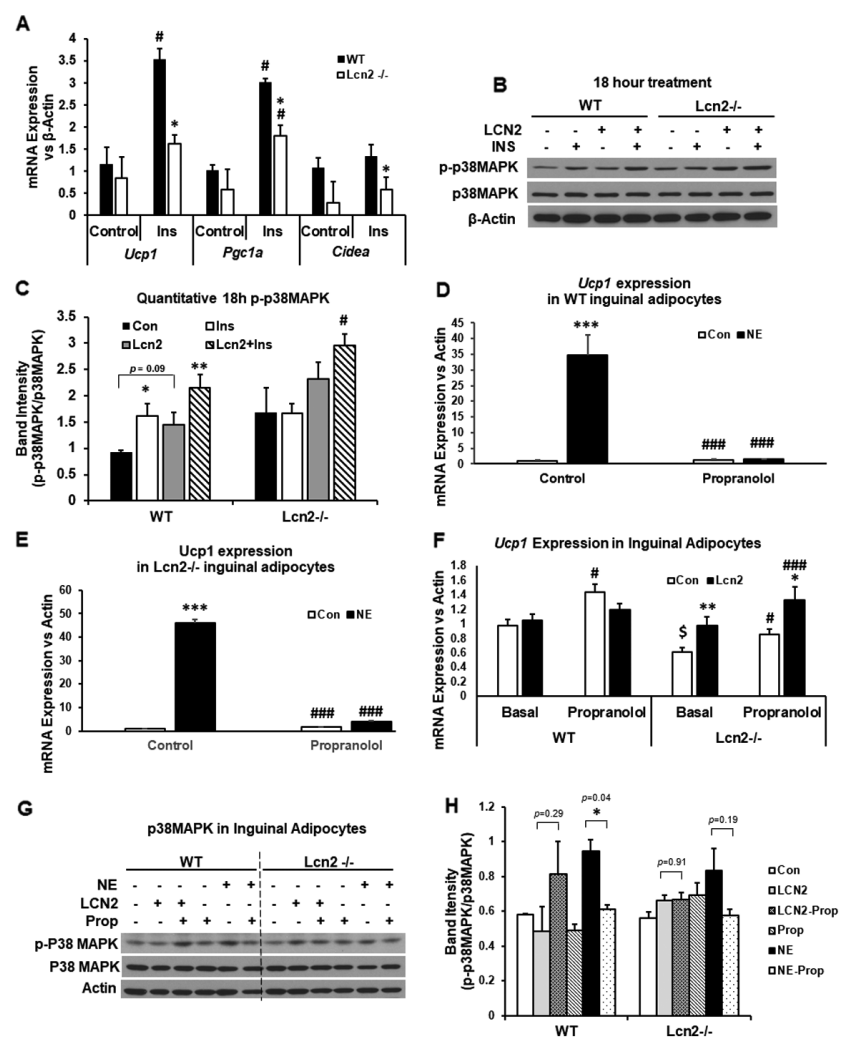

Figure 3

Thermogenic gene expression and p38MAPK signaling by recombinant LCN2 in Lcn2-l- inguinal adipocytes. (A) Gene expression of Ucp1, Pgc1a, and Cidea following $18 \mathrm{~h}$ treatment with or without insulin (INS), $n=3$ / group. (B) p38MAPK phosphorylation in differentiated inguinal adipocytes following $18 \mathrm{~h}$ of treatment with $500 \mathrm{ng} / \mathrm{mL}$ recombinant LCN2 \pm insulin (INS). (C) Quantification of band intensity of phosphorylated p38MAPK normalized to total p38MAPK for $18 \mathrm{~h}$ treatment, $n=3$ independent experiments. (D, E) Gene expression of Ucp1 following $18 \mathrm{~h}$ treatment with or without $1 \mu \mathrm{M}$ norepinephrine (NE) in the presence or absence of $100 \mu \mathrm{M}$ Propranolol, $n=3 /$ group. (F) Gene expression of Ucp 1 following $18 \mathrm{~h}$ treatment with or without $500 \mathrm{ng} / \mathrm{mL}$ recombinant LCN2 in the presence or absence of $100 \mu \mathrm{M}$ Propranolol, the data are mean from three independent experiments, $n=3$ /treatment for each experiment. (G) p38MAPK phosphorylation following $18 \mathrm{~h}$ treatment with or without $500 \mathrm{ng} / \mathrm{mL}$ recombinant LCN2 in the presence or absence of $100 \mu \mathrm{M}$ Propranolol. (H) Quantification of band intensity of phosphorylated p38MAPK normalized to total p38MAPK for Western blots in G, $n=2$ independent experiments. For A, B, C, D, E, F, G and H, the experiments were repeated $2-3$ times yielding similar results. The data are represented as mean \pm S.E.M. ${ }^{*} P<0.05$ vs WT of same group; $* * P<0.01$ vs WT of same group; ${ }^{* *} P<0.001$ vs WT of same group; $\# P<0.05$ vs Control of same genotype; $\# P<0.01$ vs Control of same genotype; $\# \# P<0.001$ vs Control of same genotype; $\$ P<0.05$ vs WT control. adipocytes, but the effect of insulin was blunted in Lcn2-/adipocytes (Fig. 3B and C). Treatment with recombinant LCN2 for $18 \mathrm{~h}$ was able to restore phosphorylation of p38MAPK in Lcn2-/- adipocytes with insulin (Fig. 3B and C). Lastly, treatment with recombinant Lcn2 was able to increase Ucp1 gene expression in Lcn2-/- adipocytes but not WT adipocytes (Fig. 3F). Together, these data suggest that LCN2 is required for and can increase insulinstimulated p38MAPK phosphorylation and upregulation of $U c p 1$ gene expression.

To further provide the evidence supporting that Lcn2 $\mathrm{KO}$ does not affect adrenergic signaling, we determined if blocking beta-adrenoreceptor signaling using propranolol would affect the effect of recombinant LCN2 on Ucp1 gene expression and p38MAPK phosphorylation. Differentiated inguinal adipocytes were treated with LCN2 or NE in the presence or absence of propranolol for $18 \mathrm{~h}$. NE significantly upregulated $U c p 1$ gene expression (Fig. 3D and E) and p38MAPK phosphorylation (Fig. 3G and H) in both WT and Lcn $2^{-/-}$adipocytes, and propranolol completely blocked the effect of NE on Ucp1 expression (Fig. 3D and E) and p38MAPK phosphorylation (Fig. 3G and H) in both types of cells. LCN2 had no effect on Ucp1 expression and p38MAPK phosphorylation in WT adipocytes, but significantly upregulated Ucp1 expression (Fig. 3F) and increased p38MAPK phosphorylation (Fig. 3G and $\mathrm{H}$ ) in Lcn2-/- adipocytes. Interestingly, propranolol was unable to block LCN2 effect on Ucp1 expression (Fig. 3F) and p38MAPK phosphorylation (Fig. 3G and H) in both WT and $L \mathrm{cn} 2^{-/-}$adipocytes. On the contrary, propranolol enhanced LCN2 induction of Ucp1 expression in Lcn2-/adipocytes (Fig. 3F). All these results suggest that LCN2 affects $U c p 1$ expression through $\beta$-adrenergic receptor signaling-independent pathway.

\section{LCN2 deficiency impairs retinoic acid-induced beiging and thermogenesis in LCn2-/- inguinal adipocytes}

RA has previously been shown to increase UCP1 expression, lipolysis, mitochondrial function and brownlike morphology of WAT adipocytes (Alvarez et al. 1995, Kumar et al. 1999, Mercader et al. 2006). RA is known to exert its effect on thermogenesis through binding and nuclear translocation of the retinoic acid receptor-alpha (RAR- $\alpha$ ), which serves as a transcription factor for several genes including Ucp1 and Pgc1a (Alvarez et al. 1995, Rabelo et al. 1996, Teruel et al. 2003). It has previously been reported that the lipocalin family of proteins bind to hydrophobic ligands including retinoids (Flower 1996), so 
we next sought to determine whether LCN2 is involved in RA-induced beiging of WAT. As shown in Fig. 4A, B and C, chronic treatment (24 days) of 12-week HFD-fed mice with RA resulted in a significant increase in UCP1 protein levels and thermogenic gene expression in iWAT of WT mice, but this effect was completely attenuated in inguinal WAT from $L c n 2^{-1-}$ mice. In in vitro studies with differentiated inguinal adipocytes, acute (24-h) treatment of WT inguinal adipocytes with RA resulted in an upregulation of $U_{c p} 1$ gene expression, but this was blunted in adipocytes from Lcn2-/- mice (Fig. 4D). When WT inguinal adipocytes were treated with RA in the presence of insulin, a synergistic effect was observed on $U c p 1$ and $P g c 1 a$ gene expression (Fig. 4D and E), suggesting that RA requires insulin for maximal activation of thermogenic gene expression in white adipocytes. The synergistic effect between RA and insulin on gene expression was significantly attenuated in Lcn $2^{-/-}$inguinal adipocytes. Interestingly, RA was able to rapidly phosphorylate p38MAPK in WT inguinal
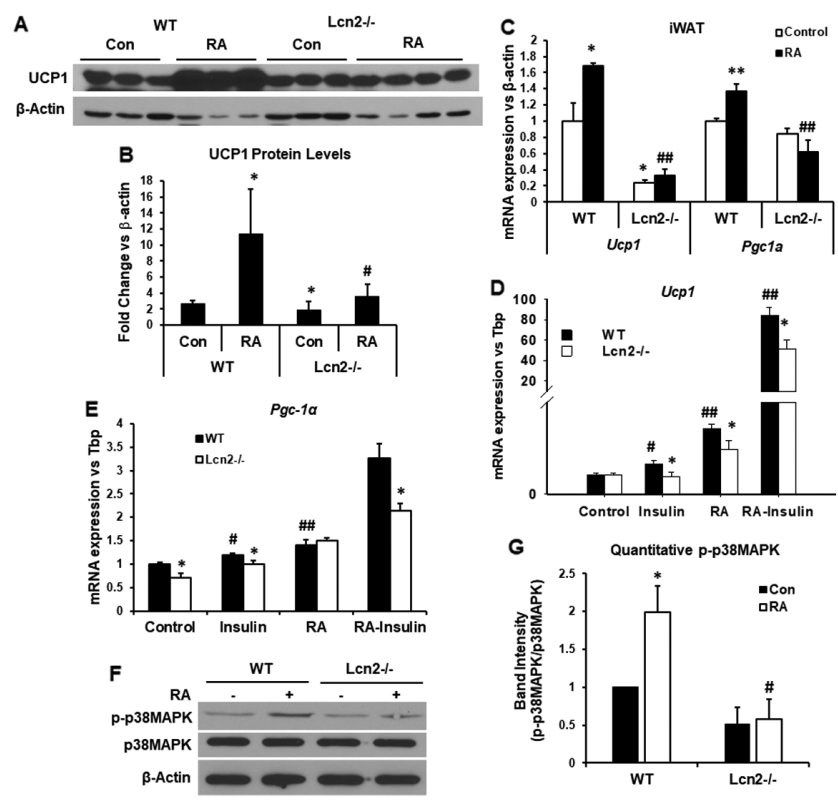

\section{Figure 4}

RA-induced thermogenesis in Lcn2-I- iWAT and adipocytes. (A, B and C) UCP1 protein levels (A and B) ( $n=3-4$ mice/group) and Ucp1 and Pgc1a gene expression (C) ( $n=4-6$ mice/group) in iWAT from WT and $L c n 2^{-/-}$ mice following oral gavage with RA for 14 days. ${ }^{*} P<0.05$ vs WT; ${ }^{\#} P<0.05$ vs WT RA. (D and E) Ucp1 (D) and Pgc1a (E) gene expression in differentiated inguinal adipocytes following $24 \mathrm{~h}$ treatment with insulin and $1 \mu \mathrm{M} \mathrm{RA}, n=3$ /treatment. ${ }^{*} P<0.05$ vs WT; $\# P<0.05$ vs WT-Con. (F and $\mathrm{G})$ p38MAPK phosphorylation in differentiated inguinal adipocytes following $30 \mathrm{~min}$ treatment with $1 \mu \mathrm{M}$ RA, $n=4$ independent experiments. For cell culture studies, experiments were repeated 2-3 times yielding similar results. The data are represented as mean \pm S.E.M. For $B$ and $C$, ${ }^{*} P<0.05$ vs RA; ${ }^{*} P<0.01$ vs RA; $\# P<0.05$ vs WT of same group; $\# P<0.01$ vs WT of same group. For D, E, and G, ${ }^{*} P<0.05$ vs WT; ${ }^{\#} P<0.05$ vs WT of same group. adipocytes, indicating that RA may have a non-genomic effect on thermogenic signaling (Fig. 4F and G). This rapid effect of RA on p38MAPK phosphorylation was abolished in $L c n 2^{-1-}$ inguinal adipocytes. These data indicate that LCN2 is involved in RA induction of thermogenic gene expression and UCP1 protein levels in inguinal white adipocytes and may regulate this by promoting the rapid phosphorylation of p38MAPK by RA.

\section{LCN2 deficiency impairs activation, but not recruitment, of beige adipocytes in Lcn2-/- inguinal adipocytes}

While Lcn2-/- inguinal adipocytes have reduced thermogenic signaling and gene expression in response to insulin and RA, it is possible that impaired recruitment of UCP1+ beige adipocytes is driving the decrease in thermogenic activation induced by RA in Lcn $2^{-1-}$ mice. The transcription factor PPAR $\gamma$ has previously been shown to be involved in brown adipocyte differentiation and beiging of WAT (Petrovic et al. 2010, Ohno et al. 2012). In WT and Lcn2-/- inguinal adipocytes differentiated with or without rosiglitazone, we found similar levels of lipid accumulation, which indicates equivalent rates of differentiation (Fig. 5A). Further, expression of adipogenic genes and PPAR $\gamma$ protein levels were similar between WT and Lcn2-/- adipocytes (Fig. 5B, D and F). To test the effect of rosiglitazone on recruitment of beige adipocytes, we next treated WT and Lcn2-/- adipocytes with rosiglitazone during differentiation followed by an acute stimulation of the fully differentiated adipocytes with RA. Treatment of inguinal adipocytes with rosiglitazone during differentiation results in similar Ucp1 gene expression in WT and Lcn2-/- inguinal adipocytes indicating normal recruitment of beige adipocytes in response to PPAR $\gamma$ activation (Fig. 5C). However, rosiglitazone during differentiation was not able to rescue the acute effect of RA on Ucp1 gene expression. Likewise, rosiglitazone during differentiation induces similar levels of UCP1 protein in WT and Lcn2-/- inguinal adipocytes, while the acute addition of RA for $24 \mathrm{~h}$ results in a further marked increase in UCP1 protein levels in WT, but this increase was attenuated in Lcn2-/- adipocytes (Fig. 5D and E). This indicates that while the recruitment of beige adipocytes and thermogenic capacity by rosiglitazone is intact, thermogenic activation of beige adipocytes by RA is impaired in Lcn2-/inguinal adipocytes.

We saw a synergistic effect between RA and insulin on thermogenic gene expression, leading us to question whether the insulin signaling pathway is involved in regulation of thermogenic activation. Indeed, activation 


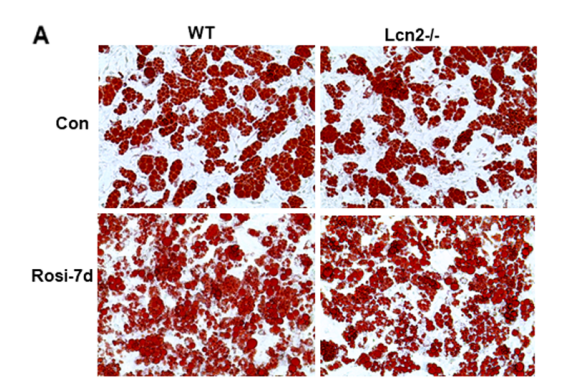

B
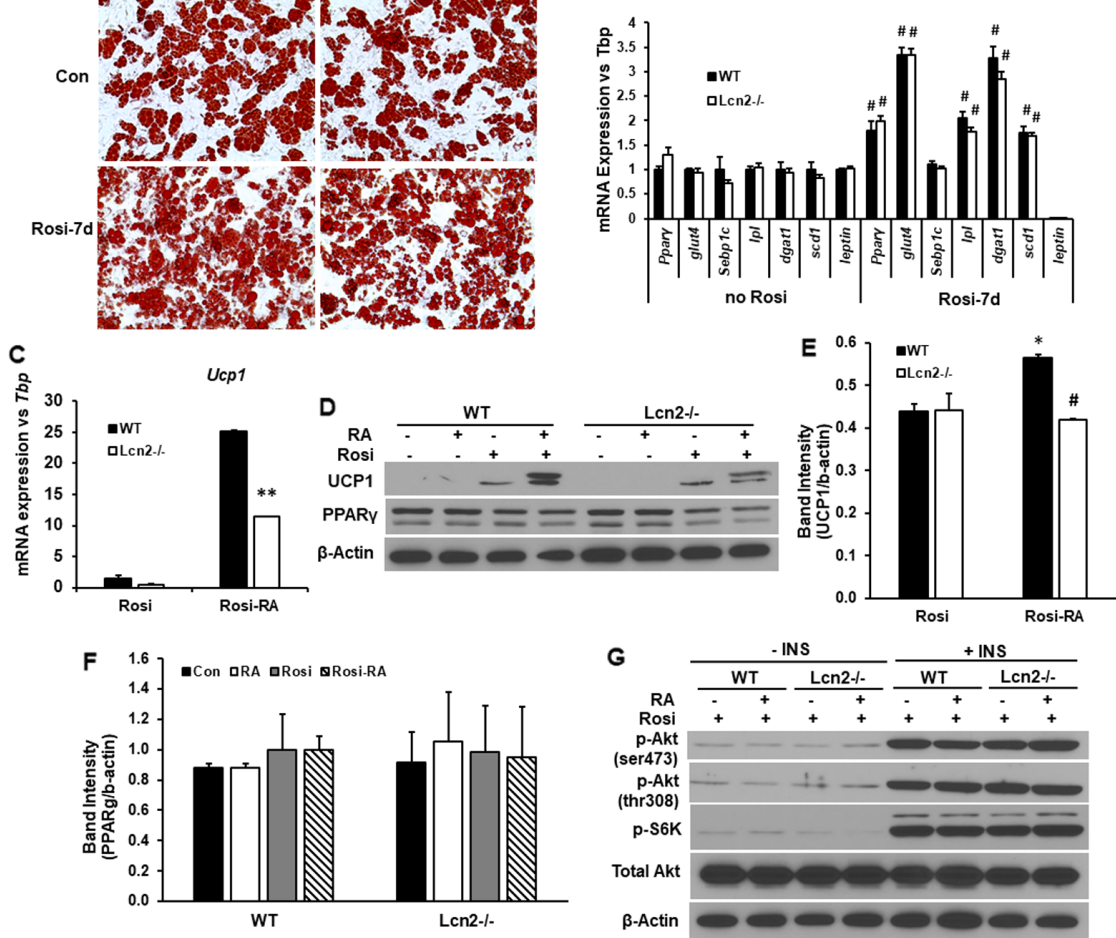

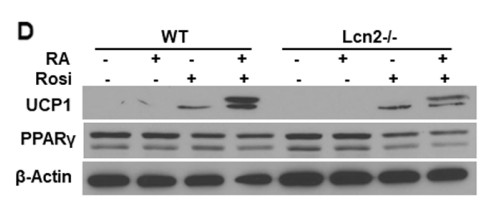

G

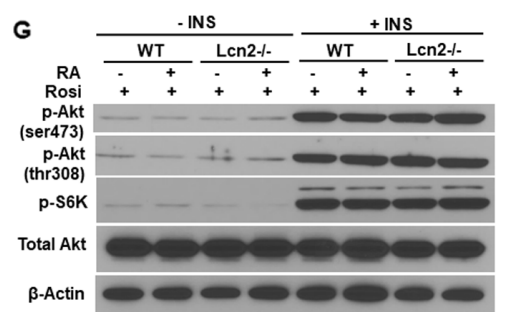

\section{Figure 5}

Effect of rosiglitazone on thermogenesis in Lcn2-l- inguinal adipocytes. (A) Oil red O Staining in differentiated inguinal adipocytes treated with or without $1 \mu \mathrm{M}$ rosiglitazone (Rosi-7d) during the 7 days of differentiation to mature adipocytes. (B) Adipogenic gene expression in differentiated inguinal adipocytes treated with or without $1 \mu \mathrm{M}$ rosiglitazone (Rosi-7d) during the 7 days of differentiation to mature adipocytes, $n=3 /$ treatment. (C and D) Ucp1 gene expression (C) and protein levels (D) in differentiated inguinal adipocytes treated with 7 days of $1 \mu \mathrm{M}$ rosiglitazone (Rosi) during differentiation followed by $24 \mathrm{~h}$ treatment with $1 \mu \mathrm{M}$ RA, $n=3$ / treatment. (E) Quantification of band intensity of UCP1 protein for western blots in D. (F) Quantification of band intensity of PPAR $\gamma$ for western blots in D. (G) Levels of proteins involved in insulin signaling in differentiated inguinal adipocytes treated with $1 \mu \mathrm{M}$ rosiglitazone (Rosi) during the 7 day differentiation to adipocytes followed by $1 \mu \mathrm{M}$ RA or insulin for $30 \mathrm{~min}$. Experiments were repeated $2-3$ times yielding similar results. The data are represented as mean \pm S.E.M. ${ }^{*} P<0.01$ vs WT from similar treatment group; ${ }^{\#} P<0.05$ vs no rosi treatment; $\# \#<0.01$ vs no rosi treatment. A full color version of this figure is available at https://doi. org/10.1530/JME-18-0017. of mammalian target of rapamycin complex 1 (mTORC1) has recently been found necessary for $\beta$-adrenergic beiging of WAT (Liu et al. 2016). Therefore, we hypothesized mTORC1 activation by insulin may be required to fully activate RA-induced beiging and that defects in insulin signaling in Lcn2-/- inguinal adipocytes could explain the differences in response to insulin and RA in these cells. Inguinal SV cells from WT and Lcn2-/- mice were differentiated to adipocytes with rosiglitazone, followed by 30 -min treatment with RA in the presence or absence of insulin. However, there were no differences in phosphorylation of Akt or ribosomal protein S6 kinase (S6K) between WT and Lcn2-/- rosiglitazone-recruited beige adipocytes in response to 30-min insulin stimulation (Fig. 5G). Further, 30-min RA treatment did not increase phosphorylation of S6K with or without the presence of insulin, suggesting mTORC1 activity does not mediate the effects of RA on beiging. This suggests LCN2 regulates the synergistic effect of insulin and RA on thermogenic gene expression through a mechanism independent from insulin-mTORC1 signaling activity.

\section{LCN2 is required for insulin-induced RAR-a translocation to the plasma membrane}

As we saw rapid phosphorylation of p38MAPK by RA, we next pursued non-genomic mechanisms by which RA and insulin could synergistically regulate thermogenesis. A pool of RAR- $\alpha$ in plasma membrane lipid rafts has previously been reported to facilitate RA action on p38MAPK in mouse embryonic fibroblasts (Piskunov \& RochetteEgly 2012, Al Tanoury et al. 2013). LCN2 is a secreted protein, which has been shown to localize to the plasma membrane and has been speculated to be involved in lipid raft rearrangement (Lingwood 2014, Watanabe et al. 2014). Additionally, our group has previously shown normal translocation of RAR- $\alpha$ to the nucleus and normal induction of RA-responsive non-thermogenic genes in Lcn2-/- adipocytes (Guo et al. 2016), further suggesting a non-genomic regulation of RA signaling by LCN2. Thus, we hypothesized that LCN2 may localize to the plasma membrane and regulate RAR- $\alpha$ translocation to the plasma membrane. Differentiated brown and inguinal adipocytes were treated with insulin or insulin plus RA for $45 \mathrm{~min}$, followed by isolation of the plasma membrane fraction. As expected, we found abundant LCN2 in plasma membrane fractions isolated from brown and inguinal adipocytes from WT mice, and insulin treatment enhanced plasma membrane abundance of LCN2 (Fig. 6A). Interestingly, RAR- $\alpha$ translocation to the plasma membrane was also significantly increased in response to insulin stimulation in WT inguinal and brown adipocytes (Fig. 6A, B and D). Insulin-stimulated RAR- $\alpha$ translocation was not significant in Lcn $2^{-/-}$inguinal adipocytes, suggesting LCN2 mediates 
this effect (Fig. 6B and D). Interestingly, addition of RA to insulin led to a reduction in plasma membrane RAR$\alpha$ in inguinal adipocytes (Fig. 6B). To understand if this RA-induced reduction in plasma membrane RAR- $\alpha$ is related to changes in overall RAR- $\alpha$ protein levels and also if LCN2 deficiency affects overall levels of RAR- $\alpha$ protein expression, we looked at the effect of RA on the mRNA and protein expression levels of RAR- $\alpha$ in WT and Lcn2-/- inguinal adipocytes with or without rosiglitazone induction during differentiation. As shown in Fig. 6C and E, mRNA and protein expression levels of RAR- $\alpha$ were not significantly different between WT and Lcn2-I- inguinal adipocytes under the basal and RA-treated conditions. RA treatment for $24 \mathrm{~h}$ resulted in a similar reduction in RAR- $\alpha$ protein levels in WT and Lcn2-/- inguinal adipocytes (Fig. 6E and F). Together, these data suggest that RA-induced decrease in plasma membrane RAR- $\alpha$ likely results from decreased total RAR- $\alpha$ protein levels.
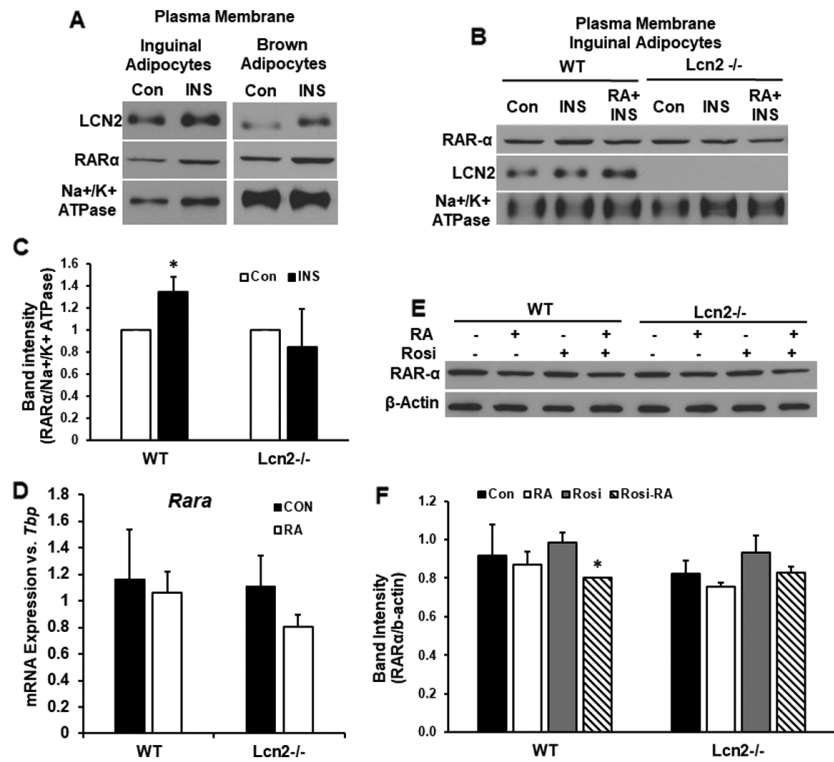

Figure 6

Plasma membrane localization of RAR- $\alpha$ in $L c n 2^{---}$brown and inguinal adipocytes. (A) Levels of RAR- $\alpha$ and LCN2 in plasma membrane isolated from WT brown adipocytes and WT inguinal adipocytes following $45 \mathrm{~min}$ treatment with insulin. (B) Levels of RAR- $\alpha$ and LCN2 in plasma membrane isolated from WT and $L C n 2^{--}$inguinal adipocytes following 45 min treatment with insulin $\pm 1 \mu \mathrm{M}$ RA. (C) Rara gene expression in differentiated inguinal adipocytes treated with $24 \mathrm{~h}$ of $1 \mu \mathrm{M} \mathrm{RA}, n=3 /$ treatment. (D) Quantification of basal and insulin-stimulated levels of RAR- $\alpha$ in plasma membrane isolated from WT and $L c n 2^{-l-}$ adipocytes following $45 \mathrm{~min}$ treatment \pm insulin, $n=4-5$ independent experiments. ( $E$ ) RAR- $\alpha$ protein levels in differentiated inguinal adipocytes treated with $1 \mu \mathrm{M}$ rosiglitazone during the differentiation to adipocytes followed by $24 \mathrm{~h}$ treatment with $1 \mu \mathrm{M}$ RA $(\mathrm{F})$. The data are represented as mean \pm S.E.M. $* P<0.05$ vs control.

\section{Discussion}

Thermogenic adaptation in BAT in response to cold is correlated with decreased BMI and improved metabolic health (Saito et al. 2009, van Marken Lichtenbelt et al. 2009, Harms \& Seale 2013). In response to thermogenic stimuli, WAT additionally recruits brown-like, 'beige' adipocytes that are multilocular, have more mitochondria and express higher levels of UCP1 (Wu et al. 2012, Harms \& Seale 2013). Our lab has previously found that LCN2 regulates thermogenesis in BAT via a non-adrenergic mechanism (Guo et al. 2010, Zhang et al. 2014b). In this study, we sought to determine whether and how LCN2 regulates beiging of WAT including beige adipocyte recruitment and activation.

Although $\beta$-adrenergic signaling facilitated by catecholamines binding to adrenergic receptors on the surface of adipocytes is the primary pathway regulating adipose tissue thermogenesis (Cao et al. 2001, 2004, Harms \& Seale 2013), alternative mechanisms independent of $\beta$-adrenergic stimulation have been reported. Insulin upregulates $U c p 1$ gene expression through activation of P13K/Akt signaling in brown adipocytes and is known to have a permissive role in mediating diet-induced thermogenesis (Rothwell \& Stock 1981, Valverde et al. 2003). Mice lacking insulin receptors in adipose tissue cannot maintain their body temperature (Boucher et al. 2012). In an early study of insulin role in BAT, knocking out insulin receptor specifically in BAT profoundly impairs brown fat adipogenesis, leading to an age-dependent loss of interscapular brown fat in mice (Guerra et al. 2001). These studies indicate insulin action is necessary for the development of thermogenic adipose tissue and thermogenesis. Additionally, insulin has been shown to play a critical role in mitochondrial function by regulating mitochondrial protein synthesis, oxidative capacity and PGC-1 $\alpha$ (McKee \& Grier 1990, Stump et al. 2003, Cunningham et al. 2007). However, the role of insulin in adipocyte thermogenesis, particularly with regard to the signaling pathway and mechanism that mediates insulin action, remains largely unexplored. LCN2 expression is highly inducible in response to insulin (Zhang et al. 2014a); nonetheless, the role of LCN2 in thermogenesis under these conditions is not well elucidated. We found LCN2 deficiency impairs p38MAPK signaling, thermogenic gene expression, and mitochondrial function in Lcn2-/adipocytes. On the other hand, recombinant LCN2 can increase p38MAPK phosphorylation and upregulate $U c p 1$ gene expression in a $\beta$-adrenergic-independent pathway as $\beta$-adrenergic receptor blocker failed to inhibit the 
effect of LCN2. This suggests that in response to insulin stimulation, LCN2 is increased and may in turn mediate insulin-permissive action on thermogenic signaling and gene expression.

Chronic activation of PPAR $\gamma$ by rosiglitazone is known to recruit UCP1+ adipocytes, particularly in iWAT (Petrovic et al. 2008, 2010, Ohno et al. 2012). Insulin is known to positively regulate $\operatorname{PPAR} \gamma$ through increasing Akt-mTORC1 pathway activity and mTORC1 activation has been reported as necessary for beiging (Kim \& Chen 2004, Liu et al. 2016). Together this suggests that insulin pathway activation might regulate WAT thermogenesis in WT mice in part by promoting PPAR $\gamma$ activity. Our lab has previously demonstrated a decrease in insulin-stimulated Akt phosphorylation in Lcn2-/- adipocytes (Guo et al. 2010), suggesting LCN2 promotes insulin pathway activation. However, it is not known whether LCN2 is necessary for insulin signaling following rosiglitazone treatment. Our results from the present study are consistent with our published studies showing that rosiglitazone treatment was able to rescue cold intolerance and UCP1 expression in BAT in Lcn2-/mice (Jin et al. 2011). Interestingly, Lcn2-/- rosiglitazonedifferentiated inguinal adipocytes remained unresponsive to acute treatment with insulin plus RA in regards to thermogenic gene expression, indicating that PPAR $\gamma$ is not involved in the acute regulation of thermogenic signaling by LCN2. Moreover, we did not observe induction of Akt and S6K phosphorylation in response to RA in both WT and Lcn2-/- rosiglitazonedifferentiated inguinal adipocytes. This further supports insulin-stimulated LCN2 secretion may regulate thermogenic signaling through mTORC1-independent mechanisms.

In addition to enhancing mitochondrial function to maximize thermogenic potential (McKee \& Grier 1990, Cunningham et al. 2007), insulin has been shown to augment the action of other thermogenic factors, including RA (Chen et al. 2009). RA synergizes with insulin to dramatically induce UCP1 expression in brown adipocytes, as well as sterol regulatory element-binding protein target gene glucokinase $(G c k)$ in hepatocytes (Chen et al. 2009, Hernandez et al. 2011). However, the molecular mechanisms and signaling pathways that link the synergistic action of insulin and RA to thermogenesis remain largely unknown. We saw a synergistic effect on thermogenic gene expression when WT inguinal adipocytes were treated with a combination of insulin and RA, and this was intriguingly blunted in Lcn2-/adipocytes.
RA has been reported to increase thermogenesis independently of $\beta$-adrenergic signaling. RA directly binds to and assists the formation of RAR $\alpha / R X R$ heterodimers, which translocate to the nucleus and increase transcription of UCP1 (Alvarez et al. 1995, Rabelo et al. 1996, Bonet et al. 1997, Mercader et al. 2010). Further studies have found RA may activate thermogenesis upstream of transcription by activating non-genomic RAR $\alpha$ signaling pathways, in particular p38MAPK (Teruel et al. 2003, Mercader et al. 2010, Al Tanoury et al. 2013). In mouse embryonic fibroblasts, RA treatment has been shown to rapidly induce translocation of RAR- $\alpha$ to plasma membrane lipid rafts, enabling RAR- $\alpha$ interaction with G-coupled proteins and resulting in increased p38MAPK activity (Piskunov \& Rochette-Egly 2012). Taken together, these studies suggest that RA enhances UCP1 levels through both genomic and non-genomic mechanisms.

LCN2 shares structural similarity with the lipocalin family of proteins, members of which have been shown to bind ligands including retinoids in a hydrophobicbinding pocket common to the family (Flower 1996, Coles et al. 1999). Previous studies in our lab have shown that LCN2 regulates RA metabolism in adipose tissue but poorly binds RA in vitro (Guo et al. 2016), suggesting

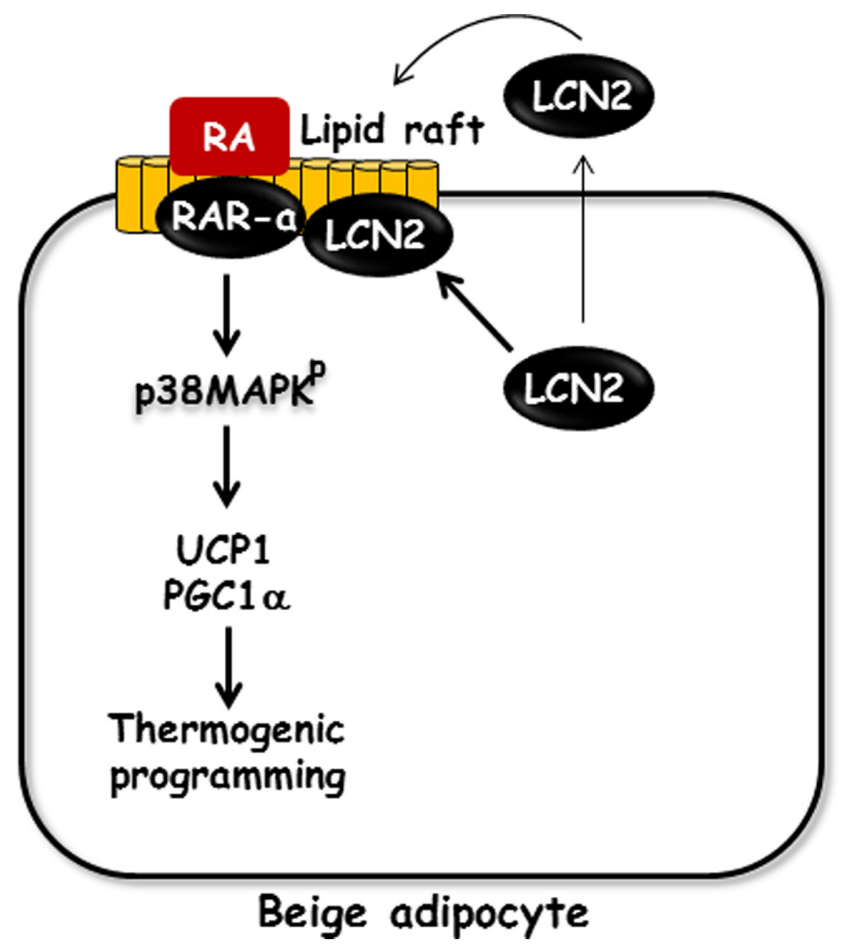

Figure 7

A model for a potential mechanism of LCN2 role in the synergistic action of insulin and retinoic acid on beiging of inguinal adipocytes. A full color version of this figure is available at https://doi.org/10.1530/JME-18-0017. 
LCN2 does not regulate RA activities by directly binding to it. Increased transcription of thermogenic genes in response to RA is known to be mediated by translocation of the nuclear transcription factor RAR- $\alpha$ from the cytosol to the nucleus (Alvarez et al. 1995, Rabelo et al. 1996, Bonet et al. 1997, Teruel et al. 2003). However, we have previously shown RA-stimulated translocation of RAR$\alpha$ to the nucleus is normal in LCN2-deficient adipocytes and not all RA-responsive genes are downregulated in the absence of LCN2 (Guo et al. 2016), suggesting a more specialized mechanism by which LCN2 regulates RA-induced thermogenic gene expression. In this study, we saw an increase in p38MAPK phosphorylation in response to a short, 30-minute treatment with RA in WT inguinal adipocytes, and this was significantly blunted in Lcn2-/- inguinal adipocytes. This indicates that LCN2 is required for RA's ability to rapidly activate a non-genomic RAR- $\alpha-$ p38MAPK pathway. LCN2 has been shown to bind to the plasma membrane of sperm and facilitate lipid raft rearrangement (Watanabe et al. 2014). Strikingly, we found both LCN2 and RAR- $\alpha$ present on the plasma membrane in brown and inguinal adipocytes. Insulin increases RAR- $\alpha$ levels on the plasma membrane in WT, but not LCN2deficient adipocytes, suggesting that LCN2 is required for insulin-stimulated plasma membrane translocation of RAR- $\alpha$. Interestingly, insulin treatment increased RAR- $\alpha$ levels to a greater extent than RA and insulin together in the plasma membrane of WT adipocytes. The reason for this is unknown, however, it may be related to degradation of RAR- $\alpha$ in response to RA-binding (Zhu et al. 1999). Indeed, we also showed that RA treatment reduces total cellular RAR- $\alpha$ protein levels in WT and Lcn $2^{-/-}$inguinal adipocytes (Fig. 6E and F).

Based on our findings, we propose a novel model where in response to insulin, LCN2 levels are increased and localize to lipid rafts on the plasma membrane (Fig. 7). Under the insulin-stimulated condition, the presence of LCN2 on the plasma membrane promotes localization of RAR- $\alpha$. This may be part of the mechanism by which RA and insulin synergistically facilitate phosphorylation of p38MAPK and Ucp1 expression. Future studies are needed to determine whether LCN2 binds intracellularly or extracellularly to the plasma membrane and how much this non-genomic RAR $\alpha$ pathway contributes to the LCN2 action on RA-induced p38MAPK pathway activation and thermogenesis. It is also unknown whether LCN2 directly regulates other unknown pathways that mediates RA effects on p38MAPK-UCP1 pathway activation. Taken together, these results show that LCN2 is a regulator of insulin and RA synergistic action on p38MAPK activation and Ucp1 gene expression by promoting localization of RAR- $\alpha$ to the plasma membrane.

Declaration of interest

The authors declare that there is no conflict of interest that could be perceived as prejudicing the impartiality of the research reported.

\section{Funding}

The project described was supported by the NIHR56 grant (DK080743) to X C, the NIDDK-funded training grant to J A D (T32DK083250), the NIDDKfunded Minnesota Obesity Center (P30DK050456), and the General Mills Foundation Chair in Genomics for Healthful Foods to X C.

\section{Acknowledgements}

The authors thank Rocio Foncea and Dr. David A. Bernlohr's group from the Department of Biochemistry, Molecular Biology and Biophysics at the University of Minnesota for technical assistance with the cellular respiration assay. This study was supported by a NIDDK Grant (R56DK080743) awarded to X C, the Minnesota Obesity Center (P30DK050456) and NIDDK-funded (T32DK083250) traineeship to J D.

\section{References}

Al Tanoury Z, Piskunov A \& Rochette-Egly C 2013 Vitamin A and retinoid signaling: genomic and nongenomic effects. Journal of Lipid Research 54 1761-1775. (https://doi.org/10.1194/jlr.R030833)

Alvarez R, de Andres J, Yubero P, Vinas O, Mampel T, Iglesias R, Giralt M \& Villarroya F 1995 A novel regulatory pathway of brown fat thermogenesis. Retinoic acid is a transcriptional activator of the mitochondrial uncoupling protein gene. Journal of Biological Chemistry 270 5666-5673. (https://doi.org/10.1074/ jbc.270.10.5666)

Bonet ML, Puigserver P, Serra F, Ribot J, Vazquez F, Pico C \& Palou A 1997 Retinoic acid modulates retinoid X receptor alpha and retinoic acid receptor alpha levels of cultured brown adipocytes. FEBS Letters 406 196-200. (https://doi.org/10.1016/S0014-5793(97)00274-3)

Boucher J, Mori MA, Lee KY, Smyth G, Liew CW, Macotela Y, Rourk M, Bluher M, Russell SJ \& Kahn CR 2012 Impaired thermogenesis and adipose tissue development in mice with fat-specific disruption of insulin and IGF-1 signalling. Nature Communications 3 902. (https:// doi.org/10.1038/ncomms1905)

Cao W, Medvedev AV, Daniel KW \& Collins S 2001 beta-Adrenergic activation of p38 MAP kinase in adipocytes: cAMP induction of the uncoupling protein 1 (UCP1) gene requires p38 MAP kinase. Journal of Biological Chemistry 276 27077-27082. (https://doi.org/10.1074/ jbc.M101049200)

Cao W, Daniel KW, Robidoux J, Puigserver P, Medvedev AV, Bai X, Floering LM, Spiegelman BM \& Collins S 2004 p38 mitogenactivated protein kinase is the central regulator of cyclic AMPdependent transcription of the brown fat uncoupling protein 1 gene. Molecular and Cellular Biology 24 3057-3067. (https://doi. org/10.1128/MCB.24.7.3057-3067.2004)

Chen X, Al-Hasani H, Olausson T, Wenthzel AM, Smith U \& Cushman SW 2003 Activity, phosphorylation state and subcellular distribution of GLUT4-targeted Akt2 in rat adipose cells. Journal of Cell Science 116 3511-3518. (https://doi.org/10.1242/jcs.00675)

Chen G, Zhang Y, Lu D, Li NQ \& Ross AC 2009 Retinoids synergize with insulin to induce hepatic Gck expression. Biochemical Journal 419 645-653. (https://doi.org/10.1042/BJ20082368) 
Coles M, Diercks T, Muehlenweg B, Bartsch S, Zolzer V, Tschesche H \& Kessler H 1999 The solution structure and dynamics of human neutrophil gelatinase-associated lipocalin. Journal of Molecular Biology 289 139-157. (https://doi.org/10.1006/jmbi.1999.2755)

Cunningham JT, Rodgers JT, Arlow DH, Vazquez F, Mootha VK \& Puigserver P 2007 mTOR controls mitochondrial oxidative function through a YY1-PGC-1alpha transcriptional complex. Nature $\mathbf{4 5 0}$ 736-740. (https://doi.org/10.1038/nature06322)

Flegal KM, Kruszon-Moran D, Carroll MD, Fryar CD \& Ogden CL 2016 Trends in obesity among adults in the United States, 2005 to 2014. JAMA 315 2284-2291. (https://doi.org/10.1001/jama.2016.6458)

Flower DR 1996 The lipocalin protein family: structure and function. Biochemical Journal 318 1-14. (https://doi.org/10.1042/bj3180001)

Guerra C, Navarro P, Valverde AM, Arribas M, Brüning J, Kozak LP, Kahn CR \& Benito M 2001 Brown adipose tissue-specific insulin receptor knockout shows diabetic phenotype without insulin resistance. Journal of Clinical Investigation 108 1205-1213. (https:// doi.org/10.1172/JCI13103)

Guo H, Jin D, Zhang Y, Wright W, Bazuine M, Brockman DA, Bernlohr DA \& Chen X 2010 Lipocalin-2 deficiency impairs thermogenesis and potentiates diet-induced insulin resistance in mice. Diabetes 59 1376-1385. (https://doi.org/10.2337/db09-1735)

Guo H, Foncea R, O'Byrne SM, Jiang HF, Zhang YY, Deis JA, Blaner WS, Bernlohr DA \& Chen XL 2016 Lipocalin 2, a regulator of retinoid homeostasis and retinoid-mediated thermogenic activation in adipose tissue. Journal of Biological Chemistry 291 11216-11229. (https://doi.org/10.1074/jbc.M115.711556)

Harms M \& Seale P 2013 Brown and beige fat: development, function and therapeutic potential. Nature Medicine 19 1252-1263. (https:// doi.org/10.1038/nm.3361)

Hernandez A, de Mena RM, Martin E \& Obregon MJ 2011 Differences in the response of UCP1 mRNA to hormonal stimulation between rat and mouse primary cultures of brown adipocytes. Cellular Physiology and Biochemistry 28 969-980. (https://doi.org/10.1159/000335810)

Jin DZ, Guo H, Bu SY, Zhang YY, Hannaford J, Mashek DG \& Chen XL 2011 Lipocalin 2 is a selective modulator of peroxisome proliferatoractivated receptor-gamma activation and function in lipid homeostasis and energy expenditure. FASEB Journal 25 754-764. (https://doi.org/10.1096/fj.10-165175)

Kim JE \& Chen J 2004 Regulation of peroxisome proliferator-activated receptor-gamma activity by mammalian target of rapamycin and amino acids in adipogenesis. Diabetes 53 2748-2756. (https://doi. org/10.2337/diabetes.53.11.2748)

Kumar MV, Sunvold GD \& Scarpace PJ 1999 Dietary vitamin A supplementation in rats: suppression of leptin and induction of UCP1 mRNA. Journal of Lipid Research 40 824-829.

Lingwood D 2014 Lipocalin 2 as a membrane-reorganizing agent. Science Signaling 7 pe19. (https://doi.org/10.1126/scisignal.2005563)

Liu D, Bordicchia M, Zhang C, Fang H, Wei W, Li JL, Guilherme A, Guntur K, Czech MP \& Collins S 2016 Activation of mTORC1 is essential for beta-adrenergic stimulation of adipose browning. Journal of Clinical Investigation 126 1704-1716. (https://doi.org/10.1172/ JCI83532)

McKee EE \& Grier BL 1990 Insulin stimulates mitochondrial protein synthesis and respiration in isolated perfused rat heart. American Journal of Physiology 259 E413-E421. (https://doi.org/10.1152/ ajpcell.1990.259.3.C413)

Mercader J, Ribot J, Murano I, Felipe F, Cinti S, Bonet ML \& Palou A 2006 Remodeling of white adipose tissue after retinoic acid administration in mice. Endocrinology 147 5325-5332. (https://doi. org/10.1210/en.2006-0760)

Mercader J, Palou A \& Bonet ML 2010 Induction of uncoupling protein-1 in mouse embryonic fibroblast-derived adipocytes by retinoic acid. Obesity 18 655-662. (https://doi.org/10.1038/ oby.2009.330)
Nedergaard J \& Cannon B 2014 The browning of white adipose tissue: some burning issues. Cell Metabolism 20 396-407. (https://doi. org/10.1016/j.cmet.2014.07.005)

Ohno H, Shinoda K, Spiegelman BM \& Kajimura S 2012 PPARgamma agonists induce a white-to-brown fat conversion through stabilization of PRDM16 protein. Cell Metabolism 15 395-404. (https://doi.org/10.1016/j.cmet.2012.01.019)

Petrovic N, Shabalina IG, Timmons JA, Cannon B \& Nedergaard J 2008 Thermogenically competent nonadrenergic recruitment in brown preadipocytes by a PPARgamma agonist. American Journal of Physiology-Endocrinology and Metabolism 295 E287-E296. (https://doi. org/10.1152/ajpendo.00035.2008)

Petrovic N, Walden TB, Shabalina IG, Timmons JA, Cannon B \& Nedergaard J 2010 Chronic peroxisome proliferator-activated receptor gamma (PPARgamma) activation of epididymally derived white adipocyte cultures reveals a population of thermogenically competent, UCP1-containing adipocytes molecularly distinct from classic brown adipocytes. Journal of Biological Chemistry 285 7153-7164. (https://doi.org/10.1074/jbc.M109.053942)

Piskunov A \& Rochette-Egly C 2012 A retinoic acid receptor RARalpha pool present in membrane lipid rafts forms complexes with $G$ protein alphaQ to activate p38MAPK. Oncogene 31 3333-3345. (https://doi.org/10.1038/onc.2011.499)

Rabelo R, Reyes C, Schifman A \& Silva JE 1996 A complex retinoic acid response element in the uncoupling protein gene defines a novel role for retinoids in thermogenesis. Endocrinology 137 3488-3496. (https://doi.org/10.1210/endo.137.8.8754778)

Rothwell NJ \& Stock MJ 1981 A role for insulin in the diet-induced thermogenesis of cafeteria-fed rats. Metabolism 30 673-678. (https:// doi.org/10.1016/0026-0495(81)90082-2)

Saito M, Okamatsu-Ogura Y, Matsushita M, Watanabe K, Yoneshiro T, Nio-Kobayashi J, Iwanaga T, Miyagawa M, Kameya T, Nakada K, et al. 2009 High incidence of metabolically active brown adipose tissue in healthy adult humans: effects of cold exposure and adiposity. Diabetes 58 1526-1531. (https://doi.org/10.2337/db090530)

Stump CS, Short KR, Bigelow ML, Schimke JM \& Nair KS 2003 Effect of insulin on human skeletal muscle mitochondrial ATP production, protein synthesis, and mRNA transcripts. PNAS $1007996-8001$. (https://doi.org/10.1073/pnas.1332551100)

Teruel T, Hernandez R, Benito M \& Lorenzo M 2003 Rosiglitazone and retinoic acid induce uncoupling protein-1 (UCP-1) in a p38 mitogenactivated protein kinase-dependent manner in fetal primary brown adipocytes. Journal of Biological Chemistry 278 263-269. (https://doi. org/10.1074/jbc.M207200200)

Valverde AM, Arribas M, Mur C, Navarro P, Pons S, CassardDoulcier AM, Kahn CR \& Benito M 2003 Insulin-induced up-regulated uncoupling protein-1 expression is mediated by insulin receptor substrate 1 through the phosphatidylinositol 3-kinase/Akt signaling pathway in fetal brown adipocytes. Journal of Biological Chemistry 278 10221-10231. (https://doi.org/10.1074/jbc. M209363200)

van Marken Lichtenbelt WD, Vanhommerig JW, Smulders NM, Drossaerts JM, Kemerink GJ, Bouvy ND, Schrauwen P \& Teule GJ 2009 Cold-activated brown adipose tissue in healthy men. New England Journal of Medicine 360 1500-1508. (https://doi.org/10.1056/ NEJMoa0808718)

Walden TB, Hansen IR, Timmons JA, Cannon B \& Nedergaard J 2012 Recruited vs. nonrecruited molecular signatures of brown, "brite," and white adipose tissues. American Journal of Physiology-Endocrinology and Metabolism 302 E19-E31. (https://doi.org/10.1152/ ajpendo.00249.2011)

Watanabe H, Takeo T, Tojo H, Sakoh K, Berger T, Nakagata N, Mak TW \& Kondoh G 2014 Lipocalin 2 binds to membrane phosphatidylethanolamine to induce lipid raft movement in a PKA-
(C) 2018 Society for Endocrinology Published by Bioscientifica Ltd. Printed in Great Britain 
dependent manner and modulates sperm maturation. Development 141 2157-2164. (https://doi.org/10.1242/dev.105148)

Wu J, Bostrom P, Sparks LM, Ye L, Choi JH, Giang AH, Khandekar M, Virtanen KA, Nuutila P, Schaart G, et al. 2012 Beige adipocytes are a distinct type of thermogenic fat cell in mouse and human. Cell $\mathbf{1 5 0}$ 366-376. (https://doi.org/10.1016/j.cell.2012.05.016)

Zhang Y, Foncea R, Deis JA, Guo H, Bernlohr DA \& Chen X $2014 a$ Lipocalin 2 expression and secretion is highly regulated by metabolic stress, cytokines, and nutrients in adipocytes. PLOS ONE 9 e96997. (https://doi.org/10.1371/journal.pone.0096997)
Zhang Y, Guo H, Deis JA, Mashek MG, Zhao M, Ariyakumar D, Armien AG, Bernlohr DA, Mashek DG \& Chen X 2014b Lipocalin 2 regulates brown fat activation via a nonadrenergic activation mechanism. Journal of Biological Chemistry 289 22063-22077. (https://doi.org/10.1074/jbc.M114.559104)

Zhu J, Gianni M, Kopf E, Honore N, Chelbi-Alix M, Koken M, Quignon F, Rochette-Egly C \& de The H 1999 Retinoic acid induces proteasome-dependent degradation of retinoic acid receptor alpha (RARalpha) and oncogenic RARalpha fusion proteins. PNAS 96 14807-14812. (https://doi.org/10.1073/pnas.96.26.14807)

Received in final form 23 May 2018

Accepted 19 June 2018

Accepted Preprint published online 19 June 2018 